\title{
COACHING: \\ UN RETO PARA LOS ORIENTADORES
}

\section{COACHING: A CHALLENGE FOR GUIDANCE PRACTITIONERS}

\author{
Rafael Bisquerra Alzina* \\ Universidad de Barcelona
}

\begin{abstract}
RESUMEN
El coaching es un tipo de asesoramiento que tiene como objetivo promover el establecimiento y la aclaración de metas, el desarrollo de competencias personales y profesionales hasta el nivel óptimo, ayudar a superar bloqueos mentales y emocionales y ayudar a llegar a ser lo mejor que uno puede ser. El coaching tiene mucho que ver con el counseling y por tanto con la orientación. Si embargo las personas que se dedican al coaching en nuestro país proceden de estudios diferentes a la orientación. Este artículo se propone llamar la atención sobre una salida profesional importante para los orientadores.
\end{abstract}

Palabras clave: coaching, counseling, consulting, mentoring, orientación psicopedagógica.

\begin{abstract}
Coaching is a type of guidance aiming to promote the establishment and explanation of goals, the development of personal and professional competencies to the optimal level, and the possibility of overcoming mental and emotional blockage and to help to achieve self-realization. Coaching is very much related with counselling. However, people engaged in coaching in our country come from studies different from guidance. This article is a call to guidance practitioners, for them to bear in mind coaching as a possible professional field.
\end{abstract}

Key words: coaching, counseling, consulting, mentoring, psycho-pedagogical guidance.

\section{Características del coaching}

El coaching es un asesoramiento que promueve el establecimiento y la aclaración de metas, objetivos y valores; el desarrollo de competencias personales y profesionales hasta el

\footnotetext{
* Catedrático de Orientación Psicopedagógica en el Departamento MIDE (Métodos de Investigación y Diagnóstico en Educación) de la Facultad de Pedagogía de la Universidad de Barcelona. Correo-e: rbisquerra@ub.edu
} 
nivel óptimo; la ayuda a superar bloqueos mentales y emocionales; la ayuda para llegar a ser lo mejor que uno puede ser y otros aspectos de desarrollo personal y profesional.

El coaching es una conversación que involucra como mínimo a dos personas. Conviene distinguir entre los tres elementos básicos del coaching:

a) coach: persona que ayuda a otra en su crecimiento personal y profesional;

b) coachee: persona que recibe la ayuda;

c) coaching: proceso de ayuda que implica al coach y al coachee. A veces el coachee puede ser un grupo.

Entre los temas del coaching están la planificación de vida, los planes de carrera, prevención y solución de problemas, el desarrollo de la inteligencia emocional, prevención del estrés, desarrollo del liderazgo entendido como relación de ayuda, etc.

En el coaching no se dice lo que uno debe hacer; pero se ayuda al interesado (coachee) a que lo descubra por sí mismo y ponga en funcionamiento los recursos necesarios para lograrlo, siempre desde su responsabilidad. Se da información sobre el comportamiento del coachee para que éste pueda mejorar sus competencias (retroalimentación correctiva). Por ejemplo, si el coachee ha llegado a la convicción de que debe desarrollar sus competencias de comunicación interpersonal, el coach le puede proporcionar feedback positivo: "Tu contacto ocular ha sido demasiado breve. Debes aumentarlo. Practica mirando directamente a la otra persona cuando estás hablando".

Para que se pueda considerar que realmente ha habido coaching debe producirse un cambio positivo en el coachee. Esto implica establecer unos objetivos del coaching y la comprobación de su logro.

Los fundamentos del coaching deben buscarse en la psicología humanista (Maslow, Rogers); el existencialismo de Sartre (libertad, decisión, responsabilidad); las aportaciones de Divine y Flaherty; el constructivismo; la PNL; influencias orientales (zen: limpiar la mente), etc.

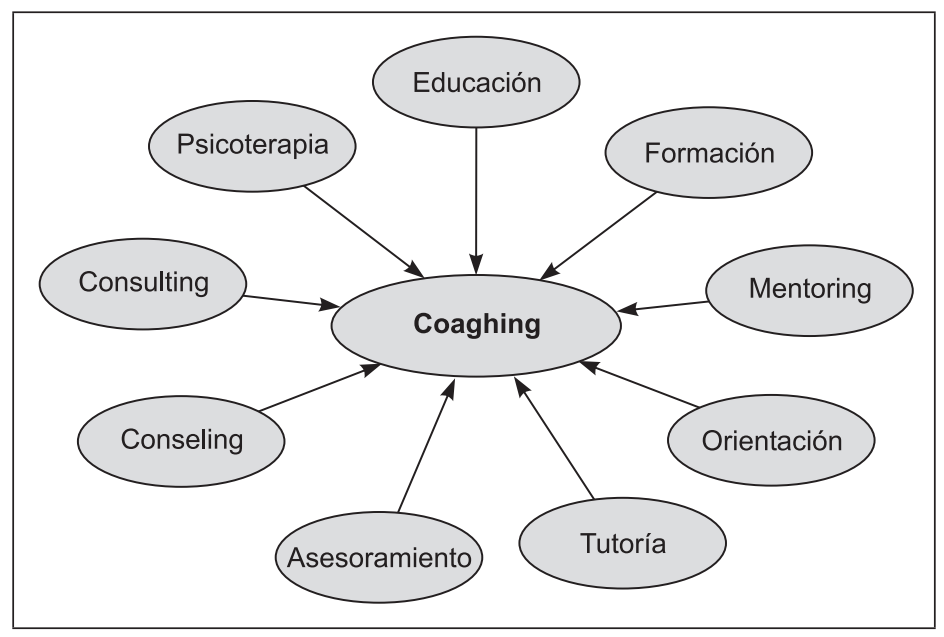


El esquema anterior pretende ilustrar los fundamentos del coaching. El esquema siguiente se propone mostrar las relaciones entre diversos conceptos interrelacionados. Por una parte, el entrenamiento (training), del cual deriva en formación y coaching. Por otra counseling, que deriva en psicoterapia a partir de los años cuarenta y que a partir de los sesenta se empieza a poner un progresivo énfasis en la prevención y el desarrollo. De esta última tendencia surgen el modelo de programas y la consultoría, que se interrelacionan con la tutoría, mentoring y asesoramiento. Todos estos conceptos tienen mucho en común con el coaching, y hay argumentos para considerar que todos ellos se incluyen en un concepto amplio de orientación psicopedagógica.

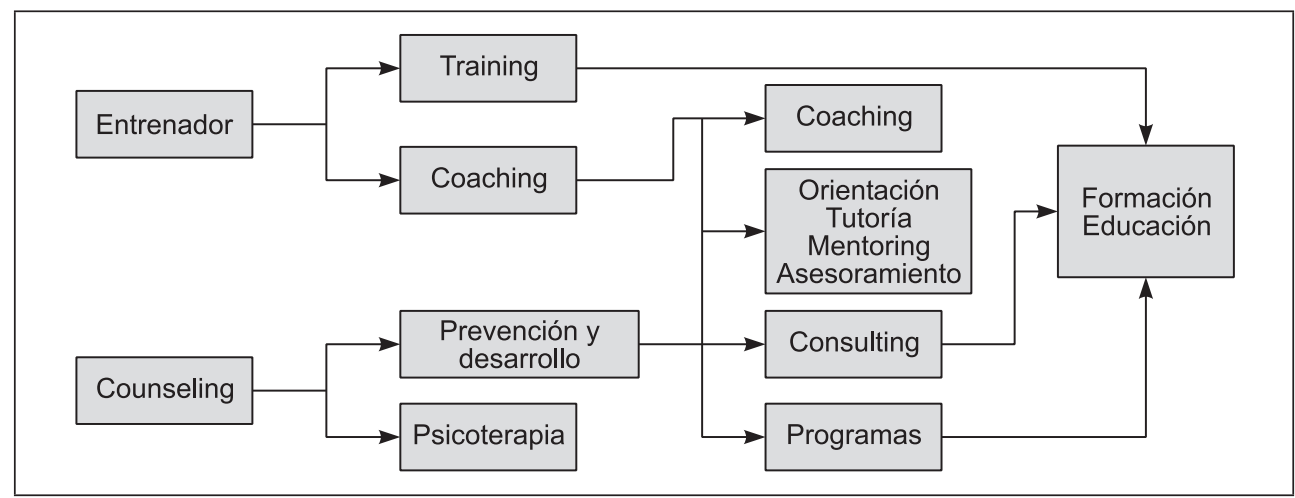

\section{Coaching y orientación}

Queda claro que coaching y orientación tienen tantos elementos en común que hay argumentos para poder considerar que el coaching es una técnica de la orientación que se ha ido abriendo camino en las empresas.

A veces se olvida o desconoce que los primeros artículos sobre coaching se publicaron en los años setenta en revistas de counseling o relacionadas con él. Ejemplos son los trabajos de Ponzo (1977) sobre el counselor-coach; Buzzotta et al. (1977) incluyen en el título de un artículo la expresión coaching \& counseling; Williamson (1986) se refiere al coaching and counseling skills; Bisquerra (1990) se refiere al coaching en un libro de orientación; posteriormente se publican abundantes trabajos que relacionan coaching y counseling; Spery (1993) relaciona counseling, consulting y coaching.

Entre los libros recomendables para los coach está La estructura de la magia, de Richard Bandler y John Grinder (1976); se considera un libro interesante para las personas que quieran mejorar sus habilidades de comunicación. También se recomiendan obras de la PNL (programación neuro-lingüística), como PNL I, El Estudio de la Estructura de la Experiencia Subjetiva de Robert Dilts, John Grinder, Richard Bandler y Judith DeLozier. Recordemos que Bandler y Grinder son dos clásicos de la orientación.

Las publicaciones especializadas de coaching aplicado a las organizaciones no empiezan hasta finales de los setenta o principios de los ochenta; véase por ejemplo Woodlands Group (1980). Pero no es hasta la década de los noventa cuando se inicia una progresiva 
difusión de manuales sobre coaching ejecutivo que llegan a constituir un auténtico "boom" editorial. Véase por ejemplo la obras de Mink, Owen y Mink (1993), Hargrove (1995), Whitmore (1996) y un largo etcétera. En la bibliografía del final de este artículo se aporta una extensa relación de obras en este sentido.

A partir de The art of Helping, de Robert R. Carkhuff (1987), una obra muy reconocida en el counseling, las autoras Mercedes Lopez y Mary Key (2006) han derivado un modelo de coaching que por su interés se reproduce a continuación, ya que integra el coaching en el counseling.

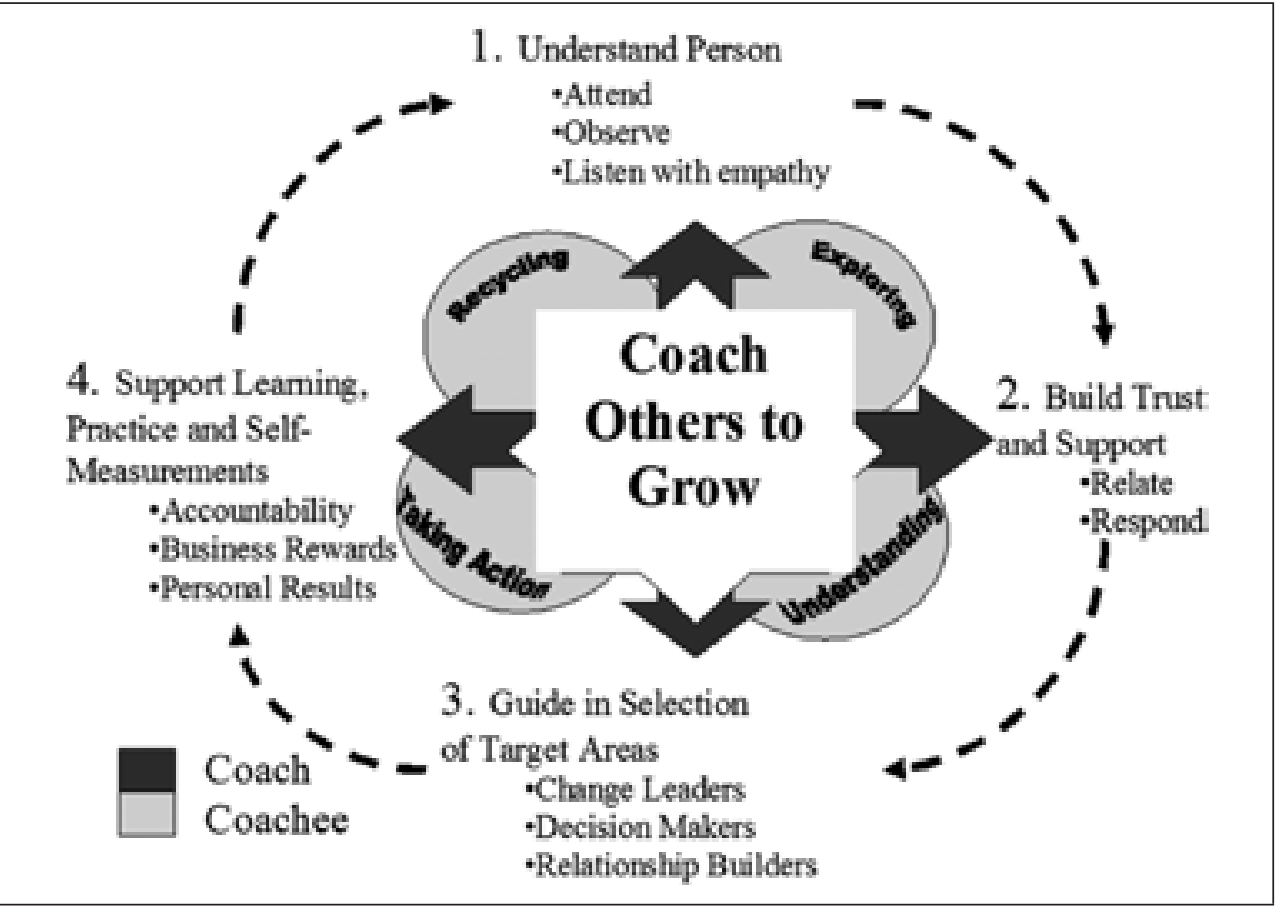

En la literatura angloamericana hay varias obras que en el título incluyen al mismo tiempo counseling y coaching. El counseling (y por extensión la orientación) es un proceso de ayuda dirigido a superar problemas personales o prevenirlos. Unos enfoques se centran más en los aspectos psicoterapéuticos y otros en la prevención y el desarrollo de competencias. Es en este último aspecto donde confluyen counseling y coaching, donde el segundo es un aspecto del primero. Podríamos decir que el coaching te ayuda a comprender como conduces un coche y cómo deberías hacerlo para conducir mejor; la orientación (counseling), desde una perspectiva más amplia, te explica las ventajas e inconvenientes de cada coche para que puedas elegir el que más te conviene; te enseña a conducir y a mejorar tu conducción.

$\mathrm{Al}$ ser un servicio que se ha desarrollado en las empresas, tiene las características de ser un proceso que muchas veces está dirigido por un gestor y que va encaminado a formar (y 
orientar) a un empleado respecto al lugar de trabajo y ayudarle a superar las barreras de cara a una óptima realización (performance) de sus funciones.

Los profesionales que se dedican al coaching han recibido una formación inicial muy diversa (economistas, abogados, directores de empresa, ingenieros, deportistas, psicólogos, lingüistas, etc.) y después se han formado en cursos sobre coaching.

Curiosamente, los pedagogos, psicopedagogos y orientadores se dedican muy poco al coaching. No abundan los trabajos publicados en revistas científicas sobre el coaching aplicado a la educación o a la orientación. Algunos ejemplos ilustrativos son los trabajos de Bowman y McCormick (2000), Griffin, Wohlstetter y Bharadwaja (2001), Mestre (2003), Slater y Simmons (2001), Syracuse University Library (2003), etc.

Coaching y counseling comparten las mismas competencias (escucha activa, empatía, preguntas abiertas, informar, planificar, ayudar, desarrollo de competencias, etc.). La identidad entre ambos procesos es tal, que fácilmente se pueden confundir, de tal forma que en la práctica uno no sabe si está haciendo counseling o coaching.

\section{El coaching como reto de futuro}

Es conocido que Monsieur Jourdain, de Le Bourgeois gentilhomme de Molière, descubrió, sorprendido, que llevaba cuarenta años hablando en prosa, sin saberlo. Igualmente nos podemos encontrar que muchos orientadores están haciendo coaching sin ser conscientes de ello. En general, hacen algún tipo de coaching en el contexto de la educación formal.

Pocas son todavía las personas formadas en orientación psicopedagógica que se atrevan a dedicarse al coaching en las organizaciones. ¿A qué es esto debido? Hay muchas razones. Por una parte hay que reconocer que la literatura sobre orientación en España ha hecho, hasta estos momentos, poco énfasis en el coaching, de tal forma que muchos todavía no saben ni lo que es. Esto induce a sugerir que desde la formación de orientadores se incluya el coaching como una de las posibilidades de la orientación, con una salida profesional que se está abriendo en las organizaciones y en la relación de ayuda con adultos. Para un futuro inmediato se tendrá que plantear el coaching en la educación formal.

Conviene insistir en que las personas que han recibido una formación de base sólida en coaching, aunque no sean conscientes de ello, son los orientadores. Tal vez lo que hace falta en estos momentos en insistir en ello y especificar algunos aspectos aplicables en las organizaciones.

En los últimos veinte años, la orientación se ha ido abriendo camino en las organizaciones. En la década de los ochenta se puso un énfasis especial en "el desarrollo de la carrera en las organizaciones". Todo este movimiento debería completarse con el coaching.

Sería una lástima para el futuro de la orientación, que un servicio tan directamente relacionado con nuestra práctica profesional como es el coaching, quedara solo en manos de otros profesionales. Los orientadores deben implicarse en el coaching, ya que su formación universitaria tiene mucho que ver con él.

Este es uno de los retos que se le plantean a la orientación: la formación inicial y continua de los profesionales en técnicas de coaching y su aplicación en las organizaciones, en el contexto sociocomunitario y también en la educación formal. 


\section{Resumen y conclusiones}

El objetivo de este artículo es enmarcar el coaching en el amplio marco de la orientación psicopedagógica. El coaching, en sus orígenes estuvo muy ligado al entrenamiento deportivo. Fue potenciado y difundido gracias, principalmente, a dos deportistas profesionales (Tim Gallwey y John Whitemore). Los cuales se basaron en el counseling, la psicoterapia y la psicología del deporte, para aplicarlos en el crecimiento personal y desarrollo de competencias en el contexto de las organizaciones.

Hay una serie de conceptos tan interrelacionados que se hace difícil la distinción y delimitación entre ellos. En este artículo se tata de la orientación, counseling y coaching. Pero podríamos ampliarlo a asesoramiento, tutoría, mentoring, guidance, consulting, psicopedagogía, psicoterapia, etc.

Desde una "ecología del lenguaje" hay que reconocer que ciertas palabras tienen más éxito en unos contextos que en otros. Coaching progresa en el contexto de las organizaciones; mucho menos en el contexto de la educación formal. En cambio orientación y psicopedagogía cuesta más que se introduzcan en las empresas.

Esto supone un reto para la orientación. En primer lugar, convenir en la necesidad de formación de los profesionales de la orientación en técnicas de coaching, tanto en la formación inicial como en la formación continua. Por otra parte está el reto de abrir camino en las organizaciones a las personas que se han formado en coaching desde los estudios de orientación y psicopedagogía.

En unos momentos en que se están discutiendo los nuevos planes de estudios de las nuevas titulaciones de grado y masters oficiales, en el marco del EEES (Espacio Europeo de Educación Superior), considero necesario tener presente estas consideraciones, que se presentan como un reto para el futuro de la orientación.

\section{Referencias bibliográficas}

Bandler, R., y Grinder, J. (1980). Estructura de la magia (2 vols.). Santiago de Chile: Cuatro Caminos.

Bisquerra, R. (1990). Orientación Psicopedagógica para la prevención y el desarrollo. Barcelona: Boixareu Universitaria - Marcombo.

Bisquerra, R. (1995). Origenes y desarrollo de la orientación psicopedagógica. Madrid: Narcea.

Bisquerra, R. (2000). Educación emocional y bienestar. Barcelona: Praxis.

Bisquerra, R. (Coord.). (1998). Modelos de orientación e intervención psicopedagógica. Barcelona: Praxis.

Buzzotta, V. R., Lefton, R. E., \& Sherberg, M. (1977). "Coaching and counseling: How you can improve the way it's done". Training \& Development Journal, 31(11), 50-60.

Carkhuff, R. R. (1969). Helping and Human Relations. A Primer for Lay and Professional Helpers (2 vols.). Nueva York: Holt, Rinehart \& Winston.

Carkhuff, R. R. (1971 a). The development of human resources. Nueva York: Holt, Rinehart \& Winston.

Carkhuff, R. R. (1987). The art of helping VI. Amherst, Ma.: Human Resource Development Press. 
Carkhuff, R. R. y Anthony, W. A. (1979). The skills of helping. An introduction to helping skills. Amhers, Ma.: Human Resource Development Press.

Flaherty, J. (1999). Coaching: Evoking excellence in others. Boston: Butterworth Heinemann.

Hargrove, R. (1995). Masterful coaching: Extraordinary results by impacting people and the way they think and work together. San Francisco: Jossey-Bass.

Hargrove, R. (2000). Masterful coaching fieldbook: Grow your business, multiply your profits, win the talent war! San Francisco: Jossey-Bass.

Lopez, M., y Key, M. (2006). Coaching Model. Disponible en la web: http://images.google. es/imgres?imgurl=http:// customchangesolutions.com/coachi4.gif\&imgrefurl= http:// customchangesolutions.com/coaching model.htm\& $\mathrm{h}=679 \& \mathrm{w}=901 \& \mathrm{sz}=26 \& \mathrm{hl}=$ es $\&$ start $=1 \&$ tbni d=X6df 01XHUFXbM:\&tbnh=110\&tbnw=146\&prev=/images\%3Fq\%3Dcoaching\%2BCarkhuff $\% 26$ svnum $\% 3 \mathrm{D} 10 \% 26 \mathrm{hl} \% 3 \mathrm{Des} \% 261 \mathrm{r} \% 3 \mathrm{D} \% 26 \mathrm{sa} \% 3 \mathrm{DN}$ [Consultado el 5 de agosto de 2006]

Mink, O. G., Owen, K. Q., y Mink, B. P. (1993). Developing high-performance people: The art of coaching. Reading, Massachusetts: Perseus Books.

Ponzo, Z. (1977). "Back to basics: The counsellor-coach". Canadian Counsellor, 12 (1), 55-58.

Repetto Talavera, E. (1977a). Fundamentos de orientación. La empatía en el proceso orientador. Madrid: Morata.

Rogers, C. R. (1942). Counseling and Psychotherapy. Boston: Houghton Mifflin Co. (Trad. cast.: Orientación psicológica y psicoterapia. Madrid: Narcea, 1984).

Rogers, C. R. (1951). Client-Centered Therapy. Boston: Houghton Mifflin. (Trad. cast.: Terapia centrada en el cliente. Barcelona: Paidós, 1981).

Rogers, C. R. (1961). On Becoming a Person. Boston: Houghton Mifflin. (Versión castellana: El proceso de convertirse en persona. Buenos Aires: Paidós, 1974).

Sperry, L. (1993). "Working with executives: Consulting, counseling and coaching". Individual Psychology, 49(2), 257.

Whitmore, J. (1992). Coaching for performance ( $3^{\mathrm{a}}$ ed. 2004). Londres: Nicholas Brealey.

Whitmore, J. (1996). Coaching for performance ( $2^{\text {nd }}$ ed.). London: Nicolas Brealey.

Williamson, M. G. (1986). Coaching and counselling skills. London: Library Association.

Witherspoon, R. and R. P. White (1998). Four essential ways that coaching can help executives. Greensboro, NC, Center for Creative Leadership.

Woodlands Group. (1980). "Management Development Roles: Coach, Sponsor, and Mentor". Personnel Journal, 59 (11), 918-921.

\section{Webs}

International Association of Coaches: http://www.certifiedcoach.org/

International Coach Federation: www.coachfederation.com

International Coach Federation-España: http://www.icf-es.com/

Asociación Española de Coaching: http://www.asescoaching.org 
Coaching University:

http://www.coachinc.com/CoachU/default.asp?s=1

CoachVille

http://learning.coachville.com/home/index.html

http://www.topten.org/

http://www.coachvillespain.com/Preguntas_frecuentes.htm

Código ético: The ICF (International Coach Federation) Code of Ethics:

http://www.coachfederation.org/ICF/For+Current+Members/Ethical+Guidelines/

Competencias: Coaching Core Competencies:

http://www.coachfederation.

org/ICF/For+Current+Members/Credentialing/Why+a+Credential/Competencies/

Definiciones y términos:

http://www.coachfederation.org/ICF/For+Current+Members/Credentialing/Why+a+Credential/

Portal del coaching:

http://perso.wanadoo.es/e/coaching/inner.html http://www.portaldelcoaching.com/Recursos/index.htm

Capital emocional:

http://www.capitalemocional.com/teorico.htm

Bibliografía:

http://www.asescoaching.org/bibliografia.htm

http://www.portaldelcoaching.com/Recursos/bibliografia/index.htm

Instituto de Coaching:

http://www.institutodecoaching.com/index.php?option=com_content\&task=view\&id= 6\&Itemid $=37$

Tim Gallway:

http://www.bigspeak.com/tim-gallwey.html

Thomas J. Leonard:

http://www.thomasleonard.com

Ejemplos de centros de counseling \& coaching:

http://www.coaching-counseling-center.com/

http://www.alternativefutures.com/

http://www.integritycounseling.net/

http://www.wichita-counseling.com/

http://www.marriagebuilders.com/graphic/mbi7000_counsel.html

http://www.lifeworkshelp.com/

Fecha de recepción: 27-10-2006

Fecha de revisión: 25-02-2008

Fecha de aceptación: 22-05-08 